\title{
VALOR DAS PROVAS DE POSICIONAMENTO DA PONTA DA AGULHA DE VERESS EM PUNÇÃO DO HIPOCÔNDRIO ESQUERDO NA INSTALAÇÃO DO PNEUMOPERITÔNIO
}

\author{
THE VALUE OF TESTS RESULTS FOR VERESS NEEDLE TIP PLACEMENT \\ INTRAPERITONEALLY IN THE LEFT HYPOCHONDRIUM FOR CREATING A \\ PNEUMOPERITONEUM
}

\author{
Otávio Cansanção de Azevedo ${ }^{1}$; João Luiz Moreira Coutinho Azevedo, ECBC-SP²; \\ Albino Augusto Sorbello, TCBC-SP ${ }^{3}$; Gustavo Peixoto Soares Miguel, ACBC-ES ${ }^{4}$; \\ Rodrigo Santa Cruz Guindalini, ${ }^{5}$; Antônio Cláudio de Godoy ${ }^{6}$
}

\begin{abstract}
RESUMO: Objetivo: Avaliar provas de posicionamento da agulha de Veress no hipocôndrio esquerdo na criação do pneumoperitônio. Método: Em cem pacientes puncionados no hipocôndrio esquerdo, provas de posicionamento da agulha foram avaliadas, considerando-as positivas quando, na prova da aspiração $(\boldsymbol{P} \boldsymbol{A})$, material orgânico era aspirado; na prova da resistência (PRes), exercia-se pequena força no êmbolo da seringa à infusão de líquido; na prova da recuperação $(\boldsymbol{P R e c})$, não se recobrava o líquido infundido; na do gotejamento $(\boldsymbol{P G})$, as gotas escoavam rapidamente e, na prova da pressão intraperitoneal inicial $(\boldsymbol{P P I I})$, os níveis eram $=$ 8mmHg. $\boldsymbol{P A}$ positiva denunciava iatrogenia, enquanto que $\boldsymbol{P R e s ,} \boldsymbol{P R e c , P G}$ e $\boldsymbol{P P I I}$ positivas indicavam que a ponta da agulha estava adequadamente posicionada na cavidade peritoneal. Foram calculadas a sensibilidade (S) e a especificidade (E) das provas, e os seus valores preditivos positivos (VPP) e negativos (VPN), mediante correlação dos resultados verdadeiro-positivos (a), falso-positivos (b), falso negativos (c) e verdadeiro-negativos (d), segundo as fórmulas: $\mathrm{S}=[\mathrm{a} /(\mathrm{a}+\mathrm{c})] \times 100 ; \mathrm{E}=[\mathrm{d} /(\mathrm{b}+\mathrm{d})] \mathrm{x} 100 ; \mathrm{VPP}=[\mathrm{a} /(\mathrm{a}+\mathrm{b})] \mathrm{x} 100$; VPN $=[\mathrm{d}(\mathrm{c}+\mathrm{d})] \times 100$. Resultados: Na $\boldsymbol{P} \boldsymbol{A}$, constatou-se que S e VPP não puderam ser aplicados, e $\mathrm{E}=100 \%$ e $\mathrm{VPN}=100 \%$. Na $\boldsymbol{P R e s}$, $\mathrm{S}=0 \%, \mathrm{E}=100 \%$, VPP=não existiu e $\mathrm{VPN}=90 \%$. Tanto na $\boldsymbol{P R e c}$ quanto na $\boldsymbol{P G}, \mathrm{S}=50 \%, \mathrm{E}=100 \%, \mathrm{VPP}=100 \%$ e $\mathrm{VPN}=94,7 \%$. Na PPII, obteve-se que S, E, VPP e VPN=100\%. Conclusão: Na punção no hipocôndrio esquerdo, $P A$ negativa garante ausência de lesões; a PRes é insegura quanto ao mau posicionamento da agulha, mas indica corretamente o bom; a $\boldsymbol{P R e c}$ e a $\boldsymbol{P G}$ não reconhecem bem o adequado posicionamento, mas detectam com segurança o inadequado; a PPII acusa com segurança tanto o mau quanto o bom posicionamento da agulha, sendo a prova mais confiável dentre as estudadas (Rev. Col. Bras. Cir. 2006; 33(5): 279-284).
\end{abstract}

Descritores: Laparoscopia/ efeitos adversos; Procedimentos cirúrgicos operatórios; Pneumoperitônio artificial; Punções/ métodos; Punções/ efeitos adversos.

\section{INTRODUÇÃO}

O estabelecimento do pneumoperitônio é uma das manobras mais críticas da laparoscopia ${ }^{1}$, não existindo consenso atual quanto ao melhor método de acesso à cavidade peritoneal visando à sua criação ${ }^{2}$. A técnica fechada mediante punção com agulha de Veress é a mais freqüentemente utilizada ${ }^{3,4}$, mas após a punctura e durante a insuflação do gás nem sempre fica evidente a localização exata da ponta da agulha.
A agulha de Veress foi desenvolvida em 1938 pelo médico húngaro János Veress com a finalidade de criar pneumotórax no tratamento da tuberculose $\mathrm{e}^{5,6} \mathrm{e}$ atualmente é utilizada como via de acesso à cavidade abdominal para a criação de pneumoperitônio com a finalidade de propiciar procedimentos laparoscópicos ${ }^{7}$.

Essa agulha tem a capacidade de perfurar os tecidos da parede abdominal mediante sua ponta em forma de bisel. Ao adentrar a cavidade peritoneal, cessa a resistência oferecida pelos tecidos à sua progressão, o que permite a saída do

1. Médico-Assistente do Serviço de Gastroenterologia Cirúrgica do Hospital do Servidor Público do Estado de São Paulo; Doutorando do Programa de Pós-Graduação em Cirurgia e Experimentação da Universidade Federal de São Paulo (UNIFESP). Pesquisador do Grupo de Videocirurgia do $\mathrm{CNPq} / \mathrm{UNIFESP}$.

2. Professor Adjunto Mestre e Doutor do Departamento de Cirurgia e Responsável pelo Setor de Videocirurgia da Disciplina de Técnica Operatória e Cirurgia Experimental (TOCE) da UNIFESP; Professor Orientador do Programa de Pós-Graduação em Cirurgia e Experimentação da UNIFESP; Ex-Professor Visitante da Universidade de Lyon, França; Líder do Grupo de Pesquisa em Videocirurgia do CNPq/UNIFESP.

3. Médico-Assistente do Serviço de Gastroenterologia Cirúrgica e Chefe do Setor de Videocirurgia do Hospital do Servidor Público do Estado de São Paulo; Professor Colaborador do Setor de Videocirurgia da TOCE-UNIFESP e do Programa de Pós-Graduação em Cirurgia e Experimentação da UNIFESP; Pesquisador do Grupo de Videocirurgia do CNPq/UNIFESP.

4. Doutorando do Programa de Pós-Graduação em Cirurgia e Experimentação da Universidade Federal de São Paulo (UNIFESP); Pesquisador do Grupo de Videocirurgia do CNPq/UNIFESP.

5. Aluno de Graduação da Escola Paulista de Medicina, UNIFESP

6. Diretor do Serviço de Gastroenterologia Cirúrgica do Hospital do Servidor Público do Estado de São Paulo.

Recebido em 14-04-2006

Aceito para publicação em 13-05-2006

Conflito de interesses: nenhum

Fonte de financiamento: nenhuma

Trabalho realizado no Serviço de Gastroenterologia Cirúrgica do Hospital do Servidor Público do Estado de São Paulo (HSPE) e no Setor de Videocirurgia da Disciplina de Técnica Operatória e Cirurgia Experimental (TOCE), Departamento de Cirurgia da Universidade Federal de São Paulo (UNIFESP), Programa de Pós-Graduação em Cirurgia e Experimentação. 
interior da agulha de mandril rombudo, atraumático ${ }^{8}$. Tal sistema permite eficácia e certa segurança, constituindo-se a punção da cavidade peritoneal com a agulha de Veress numa técnica fácil e rápida.

Não obstante, apesar desse dispositivo de segurança, as lesões de grandes vasos, produzidas pela agulha de Veress, são a causa principal de morte intra-operatória em procedimentos laparoscópicos ${ }^{9}$. Há relatos de lesões de grandes vasos que mostram toda a dramaticidade da situação ${ }^{10}$. Enfatiza-se a dificuldade de se fazer o diagnóstico correto da complicação, principalmente por causa da posição retroperitoneal dos vasos ${ }^{11}$.

Curiosamente, essas lesões produzidas pela introdução da agulha de Veress na linha mediana do abdome não são prerrogativas de cirurgiões inexperientes. Schafer et al..$^{12}$ constataram que, dentre 26 lesões desse tipo, apenas quatro foram produzidas por cirurgiões inexperientes (considerando-se como tal aqueles com vivência inferior a 50 laparoscopias), enquanto que $22(85 \%)$ foram causadas por laparoscopistas considerados experientes (os que já fizeram entre 51 e100 laparoscopias) ou muito experientes (com mais de 100 laparoscopias efetuadas).

Sendo assim, torna-se imperioso, durante a punção, saber com o maior grau de precisão possível qual a localização da ponta da agulha no abdome. Provas de posicionamento da ponta da agulha antes do início da insuflação são preconizadas em livros didáticos ${ }^{13-15}$, sendo necessárias pesquisas para aquilatar o real valor desses testes.

Esta pesquisa objetiva avaliar o valor de cinco provas propostas para testar o posicionamento correto da ponta da agulha de Veress na cavidade peritoneal.

\section{MÉTODO}

Esta pesquisa foi aprovada pelo Comitê de Ética em Pesquisa do Instituto de Assistência Médica ao Servidor Público Estadual (protocolo n ${ }^{\circ}$ 045/03) e pelo Comitê de Ética em Pesquisa da Universidade Federal de São Paulo (protocolo ${ }^{\circ}$ 1405/03).

Foram incluídos nesta pesquisa cem pacientes consecutivamente agendados para serem submetidos a procedimentos laparoscópicos, por um mesmo cirurgião, no Serviço de Gastroenterologia Cirúrgica do Hospital do Servidor Público do Estado de São Paulo (HSPE), maiores que 18 anos, nãoobesos (Índice de Massa Corpórea - IMC - menor que 30kg/ $\mathrm{m}^{2}$ ), com saco peritoneal não cirurgicamente violado nem histórico de peritonite pregressa.

A amostragem foi constituída por $42 \%$ de homens e $58 \%$ de mulheres. A média de idade de 53,7 anos, com desvio padrão (dp) de 13,1 anos e amplitude (ampl) entre 27 e 77 anos. O índice de massa corporal (IMC) foi, em média, $25,4 \mathrm{~kg} / \mathrm{m}^{2}$ (dp $\left.=2,4 \mathrm{~kg} / \mathrm{m}^{2} ; \mathrm{ampl}=20,6 \mathrm{~kg} / \mathrm{m}^{2}-29,7 \mathrm{~kg} / \mathrm{m}^{2}\right)$, com altura média de $1,64 \mathrm{~m}(\mathrm{dp}=0,09 \mathrm{~m} ; \mathrm{ampl}=1,45 \mathrm{~m}-1,87 \mathrm{~m})$, e peso corporal médio de $68,7 \mathrm{~kg}(\mathrm{dp}=9,8 \mathrm{~kg} ; \mathrm{ampl}=49,5 \mathrm{~kg}-90 \mathrm{~kg})$. As colecistectomias constituíram $80 \%$ das intervenções, as fundocardioplastias $9 \%$, as herniorrafias inguinais unilaterais $8 \%$ e as herniorrafias inguinais bilaterais $3 \%$.

Foi ministrado $0,1 \mathrm{mg} / \mathrm{kg}$ de midazolam trinta minutos antes do ato anestésico. A indução foi realizada com $2 \mathrm{mg} / \mathrm{kg}$ de propofol e $0,5 \mathrm{mcg} / \mathrm{kg}$ de fentanil, e a curarização com $0,5 \mathrm{mg} /$ $\mathrm{kg}$ de atracúrio. Os pacientes foram submetidos à anestesia geral com ventilação controlada mecânica. Foi introduzido um tubo orogástrico e aspirado o conteúdo do estômago.

A agulha de Veress é composta de dois tubos inseridos um no outro. O externo é mais curto e dotado de extremidade cortante, em bisel. O tubo interno é mais longo e sua extremidade romba (atraumática) é dotada de orifício lateral pelo qual transita o gás da insuflação peritoneal. Os dois tubos deslizam um sobre o outro mediante mola que se comprime quando a agulha encontra resistência tecidual à sua introdução, permitindo que o tubo interno seja escamoteado completamente para o interior do tubo externo. Quando a agulha penetra na cavidade peritoneal a mola faz propelir para fora do tubo externo a ponta romba do tubo interno.

Nesta pesquisa foi utilizada para a punção do hipocôndrio esquerdo (Figura 1), sendo a seguir realizadas provas preconizadas para determinar se a ponta do instrumento estava adequadamente situada no sentido de propiciar a insuflação da cavidade peritoneal. Os testes realizados foram, nesta ordem: a prova da aspiração $(\boldsymbol{P A})$, com aspiração da seringa de $5 \mathrm{ml}$ através da agulha de Veress, a qual era considerada positiva quando nenhum material era aspirado, e rotulada como negativa quando qualquer tipo de material se fazia presente na seringa. Prova da resistência à infusão (PRes): injeção se $5 \mathrm{ml}$ de solução fisiológica através da agulha, verificando-se moderada resistência ao fluir do líquido (prova positiva) ou, ao contrário, constatando-se importante aumento dessa resistência (prova negativa). Prova da recuperação do líquido infundido (PRec): após a infusão de $5 \mathrm{ml}$ de solução fisiológica, aspirava-se a seringa, considerando-se positiva a prova quando não se recuperava o líquido infundido, ou negativa quando todo ou parte do líquido infundido era recuperado., Prova do gotejamento $(\boldsymbol{P G})$ : após gotejamento no reservatório da agulha, observava-se o desaparecimento imediato das gotas (prova positiva) ou, ao contrário, constatava-se o acúmulo de líquido no reservatório, com subida permanen-

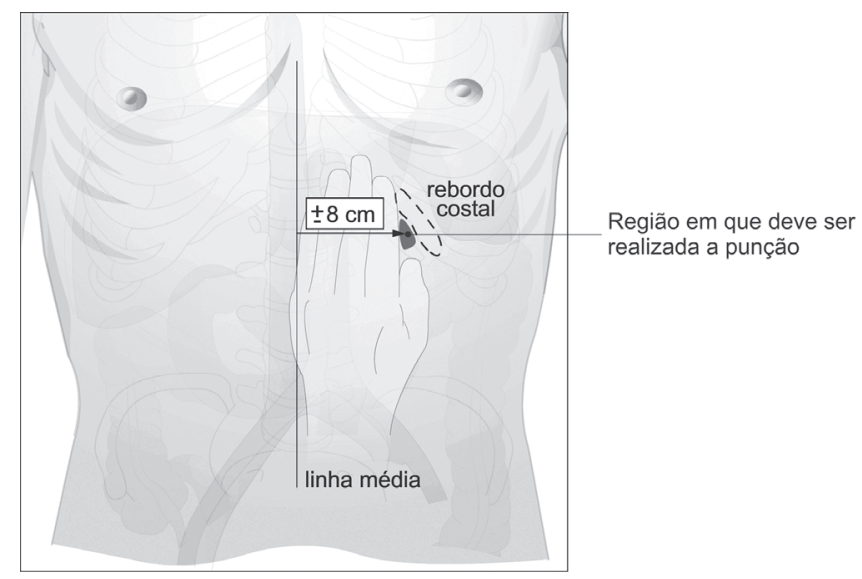

Figura 1 - Esquema representativo da localização da punção com agulha de Veress no hipocôndrio esquerdo, junto ao rebordo costal, a $8 \mathrm{~cm}$ da linha mediana. Observa-se o ponto em que deve ser realizada a punção, notando-se o distanciamento dos grandes vasos retroperitoneais. 
te da esfera da agulha (prova negativa). Prova da pressão intraperitoneal inicial (PPII): era considerada positiva (agulha em posição adequada no interior da cavidade peritoneal e com o orifício de saída de gás livre de obstrução) caso a pressão fosse igual ou menor que $8 \mathrm{mmHg}$ nos primeiros dez segundos, e considerada negativa (agulha em posição inadequada ou com obstrução do orifício da saída de gás) se fosse maior que esse valor e assim permanecesse por dez segundos.

As $\boldsymbol{P R}$ es, $\boldsymbol{P R} \boldsymbol{R}$ e $\boldsymbol{P} \boldsymbol{G}$ foram realizadas e registradas conforme o protocolo pré-determinado, uma após a outra, quer se mostrassem positivas ou negativas. Após as provas, o insuflador foi regulado para emitir um fluxo de $1,21 / \mathrm{min}$, e a pressão intraperitoneal máxima foi programada para atingir $12 \mathrm{mmHg}$. Em seguida, o insuflador foi acionado, sendo então realizada a PPII, a qual, caso fosse manifesta e permanentemente negativa, com impedimento da criação do pneumoperitônio artificial pretendido, todo o procedimento era rotulado como fracasso e a agulha de Veress era retirada da parede abdominal. O procedimento então era reiniciado. Com a PPII positiva, prosseguia-se com a insuflação de gás carbônico até a pressão atingir $12 \mathrm{mmHg}$, rotulando-se todo o procedimento como sucesso após comprovação da efetiva criação do pneumoperitônio artificial mediante a introdução da óptica laparoscópica no interior da cavidade peritoneal.

Os fracassos na tentativa de alocação da ponta da agulha de Veress no interior da cavidade peritoneal detectados por meio de cada uma das provas realizadas foram computados e tabulados.

Os resultados (positividade ou a negatividade) das provas descritas foram considerados para cálculo da sensibilidade (S), da especificidade (E), dos valores preditivos positivos (VPP) e dos valores preditivos negativos (VPN) de cada uma, segundo as fórmulas: $\mathrm{S}=[\mathrm{a} /(\mathrm{a}+\mathrm{c})] \times 100 ; \mathrm{E}=[\mathrm{d} /(\mathrm{b}+$ d) $] \times 100 ; \mathrm{VPP}=[\mathrm{a} /(\mathrm{a}+\mathrm{b})] \times 100 ; \mathrm{VPN}=[\mathrm{d} /(\mathrm{c}+\mathrm{d})] \times 100$ (Tabela 1).

A Tabela 1 evidencia os fatores envolvidos no cálculo segundo fórmulas matemáticas da sensibilidade $(\mathrm{S})$ e da especificidade (E) das provas de posicionamento da ponta da agulha de Veress realizadas após punção para a criação do pneumoperitônio, com seus valores preditivos positivos (VPP) e valores preditivos negativos (VPN).

A sensibilidade (S) das provas realizadas foi definida como a proporção dos pacientes comprovadamente portadores da condição investigada que a prova foi capaz de detectar, segundo a fórmula: $\mathrm{S}=$ [verdadeiro-positivos/(verdadeiro-positivos + falso-negativos)] x 100. A certificação de que o paciente era de fato portador da condição investigada foi conferida pela efetiva criação do pneumoperitônio artificial, diagnosticado por visão direta através de óptica laparoscópica introduzida na cavidade peritoneal. A especificidade (E) das provas foi definida como sendo a proporção dos pacientes sem a condição que foram corretamente diagnosticados como tal mediante a realização da prova, segundo a fórmula: $(\mathrm{E})=$ [verdadeiro-negativos/ (verdadeiro-negativos + falso-positivos)] x 100. A certificação de que o paciente não era de fato portador da condição foi conferida pela impossibilidade efetiva de se insuflar a cavidade peritoneal.

Foi considerado como valor preditivo positivo (VPP) a probabilidade de a agulha estar bem posicionada entre os resultados positivos de uma prova. $\mathrm{O}$ valor preditivo negativo (VPN) foi a probabilidade de a agulha estar de fato mal posicionada entre os resultados negativos de uma prova. Ambos os valores prestaram-se para a avaliação da confiabilidade dos resultados das provas, e foram calculados mediante as seguintes equações: VPP $=$ [verdadeiro-positivos/(verdadeiro-positivos + falso-positivos) $]$ x 100, e VPN = [verdadeiro-negativos/(verdadeiro-negativos + falso-negativos)] x 100.

Os dados obtidos foram tratados por análises estatísticas. As variáveis qualitativas foram representadas por freqüência absoluta e relativa e as quantitativas por média, desvio padrão e valores mínimos e máximos. Os intervalos com nível de $95 \%$ de confiança para médias e proporções foram construídos pelas fórmulas padrão para estimadores com distribuição normal.

\section{RESULTADOS}

O número máximo de tentativas para criação do pneumoperitônio em cada paciente foi de duas. Não ocorreu insuflação em local indesejado. Dos dez fracassos ocorridos, a metade foi identificada na PRec, PG $\boldsymbol{e}$ PPII, e a outra metade o foi exclusivamente na $\boldsymbol{P P I I}$ (Figura 2). A P A indicou corretamente a ausência de lesão. A PRes não foi capaz de identificar fracasso algum dos 10 ocorridos, e as $\boldsymbol{P R} \boldsymbol{R e}$ e $\boldsymbol{P} \boldsymbol{G}$ não foram capazes de identificar 5 desses dez fracassos de punções, os quais foram posteriormente identificados mediante a $\boldsymbol{P P I I}$. Na $\boldsymbol{P} \boldsymbol{A}$, constatou-se que S e VPP não puderam ser aplicados, e $\mathrm{E}=100 \%$ e VPN=100\%. Na PRes, $\mathrm{S}=0 \%, \mathrm{E}=100 \%$, VPP=não existiu e VPN=90\%. Tanto na $\boldsymbol{P R e c}$ quanto na $\boldsymbol{P G}, \mathrm{S}=50 \%$, $\mathrm{E}=100 \%, \mathrm{VPP}=100 \%$ e VPN=94,7\%. Na PPII, obteve-se que $\mathrm{S}$, E, VPP e VPN=100.

Tabela 1

\begin{tabular}{|c|c|c|c|}
\hline \multirow[t]{2}{*}{ Resultados } & \multicolumn{2}{|c|}{ Ponta da agulha em peritônio livre } & \multirow[t]{2}{*}{ Total } \\
\hline & Presente & Ausente & \\
\hline Prova positiva & $\begin{array}{l}\text { a } \\
\text { verdadeiro-positivo }\end{array}$ & $\begin{array}{l}\mathrm{b} \\
\text { falso-positivo }\end{array}$ & $\begin{array}{l}a+b \\
\text { provas positivas }\end{array}$ \\
\hline Prova negativa & $\begin{array}{l}\text { c } \\
\text { falso-negativo }\end{array}$ & $\begin{array}{l}\text { d } \\
\text { verdadeiro-negativo }\end{array}$ & $\begin{array}{l}\mathrm{c}+\mathrm{d} \\
\text { provas negativas }\end{array}$ \\
\hline Total & $\begin{array}{l}a+c \\
\text { casos positivos }\end{array}$ & $\begin{array}{l}\mathrm{b}+\mathrm{d} \\
\text { casos negativos }\end{array}$ & $\begin{array}{l}a+b+c+d \\
\text { casos }\end{array}$ \\
\hline
\end{tabular}




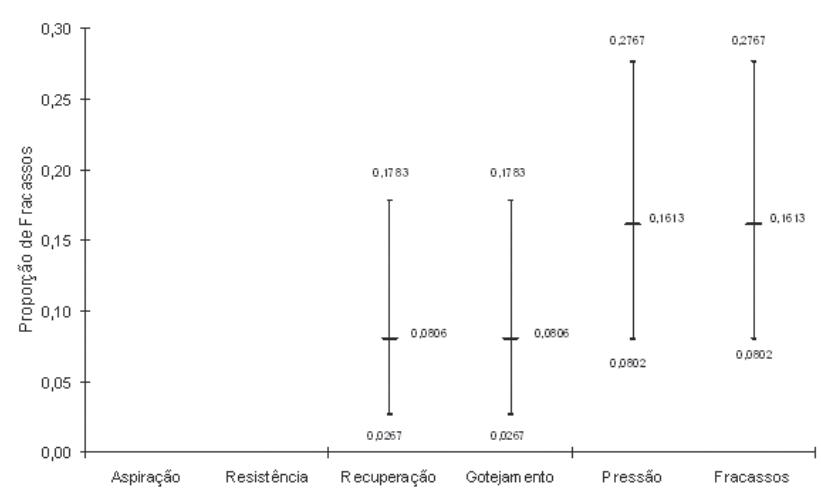

Figura 2 - Proporção dos fracassos detectados por cada uma das provas de posicionamento da ponta da agulha de Veress na cavidade peritoneal estudadas, com seus respectivos intervalos de confiança.

\section{DISCUSSÃO}

O método de criação do pneumoperitônio pela técnica fechada utilizando a agulha de Veress carece de validação objetiva das provas visando o diagnóstico da locação adequada da ponta da agulha. Há pesquisas sobre o assunto em animais de experimentação ${ }^{16}$, mas não em seres humanos.

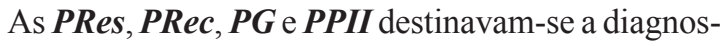
ticar uma condição específica (ponta da agulha no interior da cavidade peritoneal), sendo consideradas positivas quando os fenômenos observados faziam supor que a agulha estava nessa posição. Essas provas eram consideradas negativas quando presumivelmente a ponta da agulha se encontrava em outra localização que não em peritônio livre.

Por outro lado, a $\boldsymbol{P A}$ foi destinada especificamente a diagnosticar lesões de estruturas com a agulha logo no início do procedimento de punção, isto é, a $\boldsymbol{P} \boldsymbol{A}$ era considerada positiva quando a ponta da agulha se encontrava seguramente no interior de órgão parenquimatoso, víscera oca ou vaso sanguíneo, fato que podia ser constatado pela aspiração de fluidos ou de tecidos orgânicos. A prova da aspiração possui características peculiares na medida em que se destina a identificar a presença ou ausência de lesões e não a detectar o bom posicionamento da ponta a agulha.

Para melhor avaliação das provas procurou-se enquadrar os resultados encontrados em critérios estatísticos e matemáticos de modo a conferir credibilidade e exatidão às nossas conclusões. No contexto da detecção do posicionamento intraperitoneal da ponta da agulha de Veress, a prova ideal é aquela que, quando positiva, indica com certeza a presença da ponta da agulha na cavidade peritoneal e, quando negativa, atesta com segurança a sua ausência nesse local. Assim, as provas testadas foram analisadas quanto à sua sensibilidade (capacidade da mesma de reconhecer os verdadeiro-positivos), à sua especificidade (poder de distinguir os verdadeiro-negativos), e aos seus valores preditivos positivos (probabilidade de a agulha estar bem posicionada entre os resultados positivos da prova) e negativos (probabilidade de a agulha estar mal posicionada entre os resultados negativos da prova).
Em relação as $\boldsymbol{P R e s ,} \boldsymbol{P R e c}, \boldsymbol{P G}$ e $\boldsymbol{P P I I}$, a certificação indubitável da ocorrência de resultados verdadeiro-positivos pôde ser feita mediante a visão da ponta da agulha posicionada na cavidade peritoneal através de óptica introduzida ao término do estabelecimento do pneumoperitônio, enquanto que a validação da ocorrência de resultado verdadeiro-negativo foi realizada levando-se em conta a impossibilidade de criação do pneumoperitônio ou a realização de insuflação em local inadequado.

Esta pesquisa permitiu concluir que, na punção no hipocôndrio esquerdo com agulha de Veress para criação do pneumoperitônio artificial, a $\boldsymbol{P} \boldsymbol{A}$ negativa garante ausência de iatrogenia (excelente especificidade), a PRes é insegura quanto ao mau posicionamento da agulha, mas indica corretamente quando a ponta da agulha se encontra na cavidade peritoneal (boa sensibilidade). A $\boldsymbol{P R \boldsymbol { R } c}$ e a $\boldsymbol{P} \boldsymbol{G}$ não reconhecem bem o adequado posicionamento, mas detectam com segurança quando a ponta do instrumento não se encontra no interior do saco peritoneal (boa especificidade). A PPII acusa com segurança tanto o mau quanto o bom posicionamento da agulha (excelentes sensibilidade e especificidade), sendo a prova mais confiável dentre as estudadas.

Os principais locais de punção com agulha de Veress são a região umbilical - considerada como padrão ${ }^{16}$-, a margem subcostal esquerda ${ }^{17}$, um ponto intermediário entre a borda anterior da sínfise púbica e o umbigo ${ }^{18}$, e um ponto idêntico ao de McBurney, na fossa ilíaca esquerda ${ }^{16}$. Há, ainda, relatos de acesso à cavidade peritoneal mediante transfixação do fundo uterino com a agulha introduzida no canal cervical ${ }^{19}$ e também por meio da transfixação da parede da região tóraco-abdominal através do nono espaço intercostal esquerdo ${ }^{20}$, denominado de acesso transcostal2 ${ }^{21}$.

A preocupação com a segurança na punção às cegas com agulha de Veress na linha média, vem sendo freqüentemente motivo de estudos. Ostrzensky ${ }^{22}$ realizou estudo prospectivo, randomizado e cego, com duzentos pacientes, comparando o método tradicional de punção na linha mediana ${ }^{16}$, com uma técnica na qual a agulha de Veress era introduzida em sentido caudal na região periumbilical em angulação muito aguda em relação ao plano da parede abdominal anterior, quase paralelamente a esta. O estudo não mostrou diferenças entre quanto aos resultados das duas técnicas e enfatizou as vantagens de uma punção alternativa visando prevenir a lesão de grandes vasos ${ }^{22}$. Santala et al. ${ }^{19}$ compararam, em estudo randomizado interessando pacientes com IMC $>25 \mathrm{Kg} / \mathrm{m}^{2}$, o método tradicional, periumbilical ${ }^{16}$, com uma técnica em que a agulha de Veress era introduzida na cavidade peritoneal através do fundo uterino. A técnica transfúndica mostrou-se efetiva sem aumento do risco de hemorragia, infecção ou lesões de órgãos pélvicos. Entretanto, a técnica é contra indicada em pacientes com história de doença inflamatória pélvica, em pacientes com possibilidades de aderências infra-umbilicais, e com história de infertilidade. Este procedimento aumenta o risco teórico de endometriose e de adenomiose. Soma-se a isto o fato de só poder ser realizada em mulheres e de desagradar ao cirurgião ter que estabelecer pneumoperitônio mediante lesão de órgão $0^{23}$. 
Palmer ${ }^{17}$ descreveu a punção no hipocôndrio esquerdo com agulha de Veress a $3 \mathrm{~cm}$ abaixo do rebordo costal, na linha hemiclavicular. Desde então a técnica sofreu algumas pequenas modificações, todas visando fugir dos grandes vasos e de aderências na linha média. A punção, neste trabalho, é feita junto ao rebordo costal e a $8 \mathrm{~cm}$ da linha média (Figura 1), o que evita atingir a aorta, a veia cava e os vasos epigástricos superiores. Nessa região a punção é facilitada por ser o fixo o peritônio ao rebordo costal.

Schwartz et al. ${ }^{23}$ induziram pneumoperitônio em 600 pacientes obesos mórbidos utilizando a punção com agulha de Veress no hipocôndrio esquerdo. Ocorreu uma lesão puntiforme na camada muscular do cólon transverso, que foi reparada por laparoscopia, com um ponto simples. Não ocorreu perfuração de outras vísceras ocas, sangramento anormal de parede abdominal ou visceral, nem lesão hepática ou esplênica.

Rothagi et al. ${ }^{24}$ realizaram 344 punções no hipocôndrio esquerdo com apenas dois fracassos devido à agulha não ter se alocado na cavidade peritoneal. A única complicação foi um hematoma no grande omento, tratado com conduta expectante. Os autores concluíram que a punção no hipocôndrio esquerdo é efetiva para o estabelecimento do pneumoperitônio.

Alguns cirurgiões, habituados à punção na linha média do abdome, questionam a punção no hipocôndrio esquerdo, argumentando que essa seria mais difícil e necessitaria um maior número de tentativas para ser efetuada. Entretanto foi demonstrado que, quando comparadas, há evidências estatísticas de que as duas técnicas são equivalentes quanto às proporções de sucessos e fracassos nas tentativas de se criar o pneumoperitônio ${ }^{25}$.

Em suma, as cinco provas avaliadas nesta pesquisa demonstraram ser adequadas para orientar o cirurgião quanto ao correto posicionamento da agulha de Veress introduzida no hipocôndrio esquerdo para a criação do pneumoperitônio, contribuindo para a evitação de lesões acidentais e de insuflações equivocadas.

\begin{abstract}
Background: To evaluate tests for Veress needle tip placement intraperitoneally in the left hypochondrium for creating a pneumoperitoneum. Methods: Needle tip placement tests were evaluated in one hundred patients using the left hypochondrium area. It was considered positive when: aspiration test (PA) -returned organic material; resistance test (PRes) - a low pressure was pushed on the syringe for the liquid infusion; recovery test (PRec) - no liquid was recovered after infusion; dripping test (PG) - drops drained quickly; test for initial intraperitoneal pressure (PPII) - levels were $<=8 \mathrm{mmHg}$. Positive PA suggested bowel injury, while positive PRes, PRec, PG and PPII indicated that needle tip was adequately located in the peritoneal cavity. The Sensitivity ( $S E)$ and Specificity ( $S P$ ), as well as their predictive positive values $(P P V)$ and predictive negative values $(P N V)$ of these tests were calculated using results correlation which were true-positives (a), false-positives (b), false-negatives (c) and true-negatives (d), accordingly to the formulas: $S E=[a /$ $(a+c)] x 100 ; S P=[d /(b+d)] x 100 ; P P V=[a /(a+b)] x 100 ; P N V=[d(c+d)] x 100$. Results: If a positive PA had returned, $S E$ and $P P V$ did not fit, and $S P=100 \%$ and $P N V=100 \%$. In the PRes, $S E=0 \%, S P=100 \%, P P V=$ did not exist and $P N V=90 \%$. Both in the PRec and in the $P G$, results were for $S E=50 \%, S P=100 \%, P P V=100 \%$ and $P N V=94.7 \%$. In the PPII test results were for SE, PPV and PNV =100\%. Conclusion: Left hypochondrium negative PA guaranteed that bowel was not perforated; PRes test is a not accurate test for detection of the needle tip bad placement, however it accurately indicates its good positioning; PRec and the PG tests do not detect the adequate positioning, but they detect very well the inadequate positioning; PPII test shows with reliability both bad and good positioning of the needle, being the most trustworthy test among those studied.
\end{abstract}

Key Words: Laparoscopy/adverse effects; Surgical procedures, operative; Pneumoperitoneum, artificial; Punctures/methods; Punctures/adverse effects.

\section{REFERÊNCIAS}

1. Mouret PH. Cirugía laparoscópica: una evolución de la filosofia quirúrgica? Evaluación de los resultados de 20 años de investigación em cirurgía laparoscópica. In: Meinero M, Melotti G, Mouret PH, editores. Cirurgía laparoscópica. Madrid: Panamericana; 1996. p. 1-12.

2. Neudecker J, Sauerland S, Neugebauer E, Bergamaschi R, Bonjer HJ, Cuschieri A, Fuchs KH, Jacobi Ch, Jansen FW, Koivusalo AM, Lacy A, McMahon MJ, Millat B, Schwenk W. The European Association for Endoscopic Surgery clinical practice guideline on the pneumoperitoneum for laparoscopic surgery. Surg Endosc. 2002;16(7):1121-43. Epub 2001 May 20.

3. Molloy D, Kaloo PD, Cooper M, Nguyen TV. Laparoscopic entry: a literature review and analysis of techniques and complications of primary port entry. Aust N Z J Obstet Gynaecol. 2002;42(3):246-54.
4. Catarci M, Carlini M, Gentileschi P, Santoro E. Major and minor injuries during the creation of pneumoperitoneum. Amulticenter study on 12,919 cases. Surg Endosc. 2001;15(6):566-9. Epub 2001 Apr 3.

5. Veress J. Neues instument zur ausfuhrung von Brust-oder bauchpunktionen und pneumothoraxbehandlung. Dtsch Med Wochenshr. 1938;41(8):1480-1.

6. Bridgewater FH, Mouton WG. Rationale and intended use for the Veress needle: a translation of the original descriptive article. Surg Laparosc Endosc Percutan Tech. 1999;9(4):241-3. Erratum in: Surg Laparosc Endosc Percutan Tech 2000;10(1):57.

7. Santor J, Ballagi F, Nagy A, Rakoczi I. A needle-puncture that helped to change the world of surgery. Homage to Janos Veres. Surg Endosc. 2000;14(2):201-2.

8. Azevedo JLMC. http://www.cirurgiaonline.med.br/

9. Chandler JG, Corson SL, Way LW. Three spectra of laparoscopic entry access injuries. J Am Coll Surg. 2001;192 (4):478-90; discussion 490-1. 
10. Peterson HB, Greenspan JR, Ory HW. Death following puncture of the aorta during laparoscopic sterilization. Obstet Gynecol. 1982;59(1):133-4.

11. Pirró N, Ciampi D, Champsaur P, Di Marino V. The anatomical relationship of the iliocava junction to the lumbosacral spine and the aortic bifurcation. Surg Radiol Anat. 2005; 27(2):137-41. Epub 2004 Dec 21

12. Schafer M, Lauper M, Krahenbuhl L. Trocar and Veress needle injuries during laparoscopy. Surg Endosc. 2001;15(3):275-80. Epub 2000 Dec 12.

13. Meinero M, Melotti G, Rustichelli Gl. Entrenamiento y técnicas básicas. In: Meineiro M, Melotti G, Mouret PH, editores. Cirurgía laparoscópica. Madrid: Médica Panamericana; 1996. p. 16-27.

14. Coptcoat MJ, Coptcoat AD. General laparoscopic techniques. In: Coptcoat MJ, Coptcoat AD, editors. Laparoscopy in urology. London: Blakwell Scientific Publication; 1994. p. 28-30.

15. Nathanson LK. Instrumentos y técnicas operatórias básicas para cirurgía laparoscópica. In: Cuschieri A, Berci G, editores. Cirurgía biliar laparoscópica. London: Blakwell Scientific Publication; 1991. p. 18-19.

16. Guimarães P. Pneumoperitônio, punções e trocartes. In: Donadio $\mathrm{N}$, Albuquerque Neto LC, editores. Consenso Brasileiro em Videoendoscopia Ginecológica. São Paulo: Artes Médicas; 2001. p. 27-32.

17. Palmer R. Safety in laparoscopy. J Reprod Med. 1974;13(1):1-5.

18. Lee CL Huang KG, Jain S, Wang CJ, Yen CF, Soong YK. A new portal for gynecologic laparoscopy. J Am Assoc Gynecol Laparosc. 2001;8(1):147-50.

19. Santala M, Jarvela I, Kauppila A. Transfundal insertion of a Veress needle in laparoscopy of obese subjects: a practical alternative. Hum Reprod. 1999;14(9):2277-8.

20. Childers JM, Brzechffa PR, Surwit EA. Laparoscopy using the left upper quadrant as the primary trocar site. Gynecol Oncol. 1993;50(2):221-5.

21. Lueken RP, Busche D, Nugent W, Gallinat A, Moeller CP, Salfelder A. Transcostal access with the Veress needle in women with several previous surgeries. J Am Assoc Gynecol Laparosc. 1996;3 Suppl 4:S27.
22. Ostrzenski A. Randomized, prospective, single-blind trial of a new parallel technique of Veress pneumoperitoneum needle insertion versus the conventional closed method. Fertil Steril. 1999; 71(3):578-81.

23. Schwartz ML, Drew RL, Andersen JN. Induction of pneumoperitoneum in morbidly obese patients. Obes Surg. 2003;13:601-4; discussion 604.

24. Rohatgi A, Widdison AL. Left subcostal closed (Veress needle) approach is a safe method for creating a pneumoperitoneum. J Laparoendosc Adv Surg Tech A. 2004;14(4):278-80.

25. Azevedo OC, Azevedo JLMC, Sorbello AA, Godoy AC, Menezes FJC, Aguiar GS. Criação do pneumoperitônio mediante punção com agulha de Veress no hipocôndrio esquerdo: ensaio clínico, prospectivo e randomizado. Rev Col Bras Cir. 2005;32(5):273-8.

Como citar este artigo:

Azevedo OC, Azevedo JLMC, Sorbello AA, Miguel GPS, Guindalini RSC, Godoy AC. Valor das provas de posicionamento da ponta da agulha de veress em punção do hipocôndrio esquerdo na instalação do pneumoperitônio. Rev Col Bras Cir. [periódico na Internet] 2006 Set-Out;33(5). Disponível em URL: www.scielo.br/rcbc

Endereço para correspondência :

Prof. Dr. João Luiz M. C. Azevedo

Setor de Videocirurgia

Disciplina de Técnica Operatória e Cirurgia Experimental

Departamento de Cirurgia - Universidade Federal de São Paulo

Rua Botucatu 740 - Prédio da Cirurgia Experimental

Vila Clementino

04023-900 - São Paulo - SP

Tel: (11) 5576-4272

Email: jozevedo.dcir@epm.br

Site: http://www.cirurgiaonline.med.br 